\title{
Evolution of the distribution of stellar mass and light since redshift of unity
}

\author{
Cheng Li \\ Max Planck Institute Partner Group at Shanghai Astronomical Observatory \\ Nandan Road 80, Shanghai 200030, China \\ email: leech@shao.ac.cn
}

\begin{abstract}
We use data from the Sloan Digital Sky Survey (SDSS) and the DEEP2 survey to characterize the distribution of stellar mass and light of galaxies in the low-redshift and $z=1$ Universe. We investigate the clustering bias of stellar mass and light by comparing these to projected autocorrelations of dark matter estimated from the Millennium Simulations (MS). All of the autocorrelation and bias functions show systematic trends with spatial scale and waveband, which are impressively similar at the two redshifts. This shows that the wellestablished environmental dependence of stellar populations in the local Universe is already in place at $z=1$. The recent MS-based galaxy formation simulation of Guo et al. (2011) reproduces the scale-dependent clustering of luminosity to an accuracy better than $30 \%$ in all bands and at both redshifts, but substantially overpredicts mass autocorrelations at separations below $\sim 2 \mathrm{Mpc}$. Further comparison of the shapes of our stellar mass bias functions with those predicted by the model suggests that both the SDSS and DEEP2 data prefer a fluctuation amplitude of $\sigma_{8} \sim 0.8$ rather than the $\sigma_{8}=0.9$ assumed by the MS.
\end{abstract}

Keywords. galaxies: abundances - distances and redshifts - formation, cosmology: large-scale structure of Universe - dark matter - cosmological parameters

\section{Introduction}

In the past few years we have done a series of studies aiming at characterizing the spatial distribution of stellar mass and light in both the local Universe and the Universe at redshift $z \sim 1$. For this we have made use of galaxy samples from the Sloan Digital Sky Survey (SDSS; York et al. 2000) and the DEEP2 Galaxy Redshift Survey (Davis et al. 2003). We estimate the autocorrelation function of stellar mass or luminosity in a given band on scales larger than those of individual galaxies, by weighting each galaxy in our sample by its stellar mass or luminosity when estimating the autocorrelation function of galaxies. As we show, the autocorrelation of stellar mass or luminosity is remarkable, both for the accuracy with which it can be estimated from the current galaxy redshift surveys, and for the fact that it turned out to be almost a perfect power law over three orders of magnitude in the spatial scale. In addition, the ratio of the autocorrelation of stellar mass to that of the luminosity in a band probes the spatial distribution of stellar mass to light ratio in the Universe, while the ratio of the luminosity autocorrelation between two given bands quantifies the spatial distribution of galaxy color. Our measurements of the clustering of stellar mass and light thus provide a precise characterization of the well-known environmental dependence of stellar populations in the local Universe and its evolution up to $z \sim 1$, and are expected to provide powerful constraints on galaxy formation models.

I will briefly present the main results of our studies and the reader is referred to our papers for details (Li \& White 2009, Li \& White 2010, Li et al. 2012). 

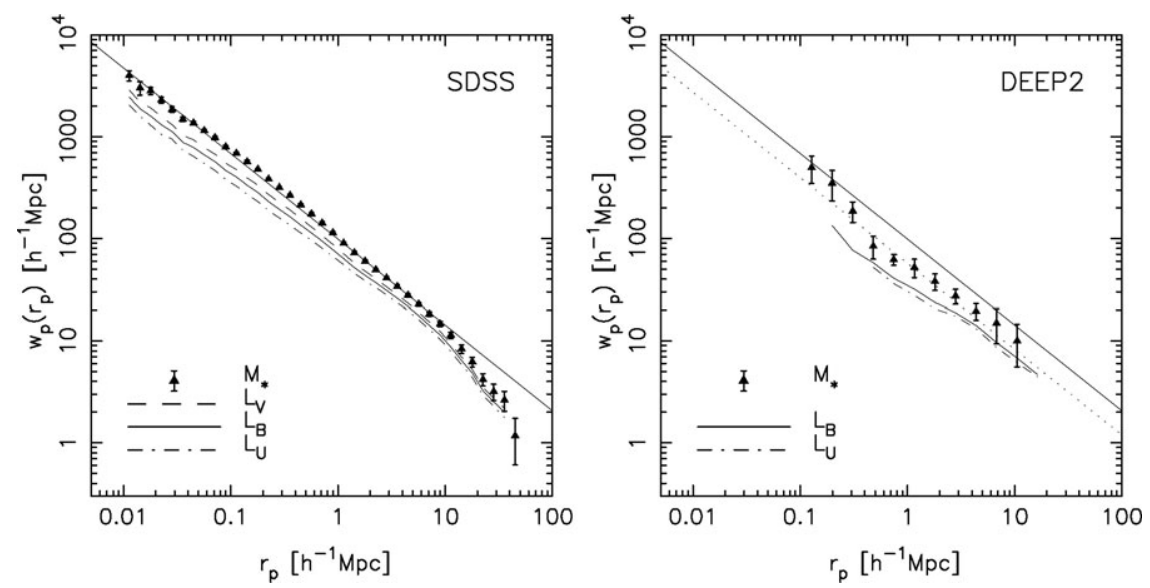

Figure 1. Projected stellar mass autocorrelation functions in the SDSS/DR7 (left panel) and in the DEEP2/DR3 (right panel) are plotted as triangles with error bars and are compared to projected luminosity autocorrelation functions measured in the rest-frame $U, B$ and $V$ bands (lines). Errors on the stellar mass autocorrelation function are estimated in the left panel from the scatter among measurements on 20 mock SDSS catalogues constructed from the Millennium Simulation, and in the right panel from the scatter between measurements on the four independent fields of the DEEP2 survey. The solid line in the left panel is a power-law fit to the SDSS data over the range $10 h^{-1} \mathrm{kpc}<r_{p}<10 h^{-1} \mathrm{Mpc}$ and is repeated in the right panel. It corresponds to a three-dimensional autocorrelation function $\xi^{*}(r)=\left(r / r_{0}\right)^{-1.84}$ with $r_{0}=6.1 h^{-1} \mathrm{Mpc}$. The dotted line in the right panel is a power-law fit to the DEEP2 data over the full range probed, with index fixed to be -1.84 , i.e. the same as what is determined for the SDSS data. The corresponding correlation length is $r_{0}=4.3 h^{-1} \mathrm{Mpc}$.

\section{Results}

Fig. 1 shows the projected autocorrelation function of stellar mass and luminosity in different bands, measured from SDSS data release 7 (DR7; left panel) and DEEP2 data release 3 (DR3; right panel). As can be seen, luminosity is less strongly clustered than stellar mass on all scales and in all wavebands. Furthermore, the amplitude and slope of the luminosity autocorrelation function changes systematically with wavelength, with the reddest band (the $V$-band) showing the highest amplitude and the steepest slope. As a result, $V$-band light clusters more similarly to stellar mass than the bluer bands shown in the figure. Finally, all the autocorrelation estimates are well described by power laws. The best-fit power law is shown in the figure only for the stellar mass autocorrelation. Power law fits for other cases are presented and discussed in detail in Li \& White (2010).

The figure also remarkably shows that the DEEP2 galaxies show very similar autocorrelation behaviors to the SDSS. Although the measurement errors are relatively large due to the smaller sample size and the considerable depth of the DEEP2, all the systematic trends seen above for the SDSS hold for the DEEP2. This shows that the well-known dependence of stellar populations on environment as seen in the local Universe is already in place at $z=1$.

In Fig. 2, we compare the autocorrelation functions measured from the two surveys with predictions of the same quantities from the recent semi-analytic model of galaxy formation of Guo et al. (2011). At both redshifts the discrepancy between model and data is clearest in the stellar mass autocorrelations. As already found by Guo et al. (2011), the projected autocorrelation of galaxies in the model exceeds that observed in the SDSS on all scales, by only $\sim 15 \%$ for scales above $\sim 2 h^{-1} \mathrm{Mpc}$, but by increasingly larger factors on smaller scales, reaching a factor of $\sim 3$ at $10 h^{-1} \mathrm{kpc}$. Remarkably, 

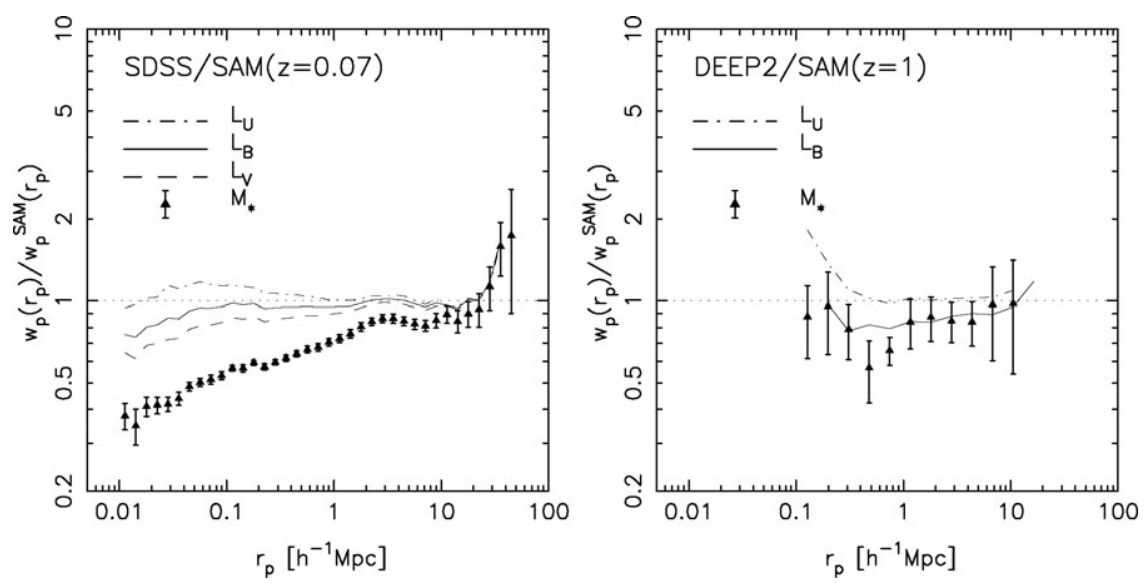

Figure 2. Ratio of the projected stellar mass (triangles) and luminosity (lines) autocorrelation functions in the SDSS/DR7 (left panel) and in the DEEP2/DR3 (right panel) to predictions of these same quantities from the semi-analytic galaxy formation simulation (SAM) of Guo et al. (2011) at $z=0.07$ (left panel) and at $z=1$ (right panel).

although the model predicts stellar mass autocorrelations that disagree with the SDSS data, its luminosity autocorrelations match the observations much better, particularly in the rest-frame $B$-band. Apparently the overly strong clustering of stellar mass is almost exactly compensated by the overabundance of passive satellite galaxies so that the light distribution is well reproduced.

At $z \sim 1$ the predicted luminosity autocorrelations lie somewhat above the DEEP2 measurements in $B$ but agree well at $U$. Given the error bars, the discrepancy at the longer wavelengths is only marginally significant. The fact that a physically based galaxy formation model can simultaneously agree with the clustering of light and disagree with that of stellar mass, yet agree with the abundance of galaxies as a function of both light and stellar mass (see G11), demonstrates the complexity of the constraints on galaxy formation implied by precise observations of abundance and clustering. The agreements and disagreements with DEEP2 data at $z \sim 1$ are very similar to those with SDSS data at low redshift, suggesting that the model is treating the evolution and clustering of the galaxy population in a realistic way.

\section{Discussion}

An interesting feature in the observed bias functions (ratios of the observed autocorrelations relative to the autocorrelation of dark matter; see fig.2 of Li et al. 2012) is an obvious step at the one-halo/two-halo transition at about $2 \mathrm{Mpc}$ which reflects the marked change in slope of the dark matter correlations at this point (see figs. 2 and 6 of Li \& White 2009). Since physically consistent models for the evolution of the dark matter and galaxy distributions show smooth bias behaviour over this separation range, this feature suggests that the amplitude of mass fluctuations is too high in the Millennium Simulation, producing a one-halo/two-halo transition which is stronger in the simulated mass distribution than in the true mass distribution. If this interpretation is correct, the shape of our bias functions can be used to estimate the value of the fluctuation amplitude parameter $\sigma_{8}$. We illustrate this possibility in Figure 3 where we plot the bias determined from the clustering of stellar mass over the separation range $200 h^{-1} \mathrm{kpc}<r_{p}<15 h^{-1} \mathrm{Mpc}$ both for the SDSS $(z=0.07)$ and for the DEEP2 $(z=1)$. 

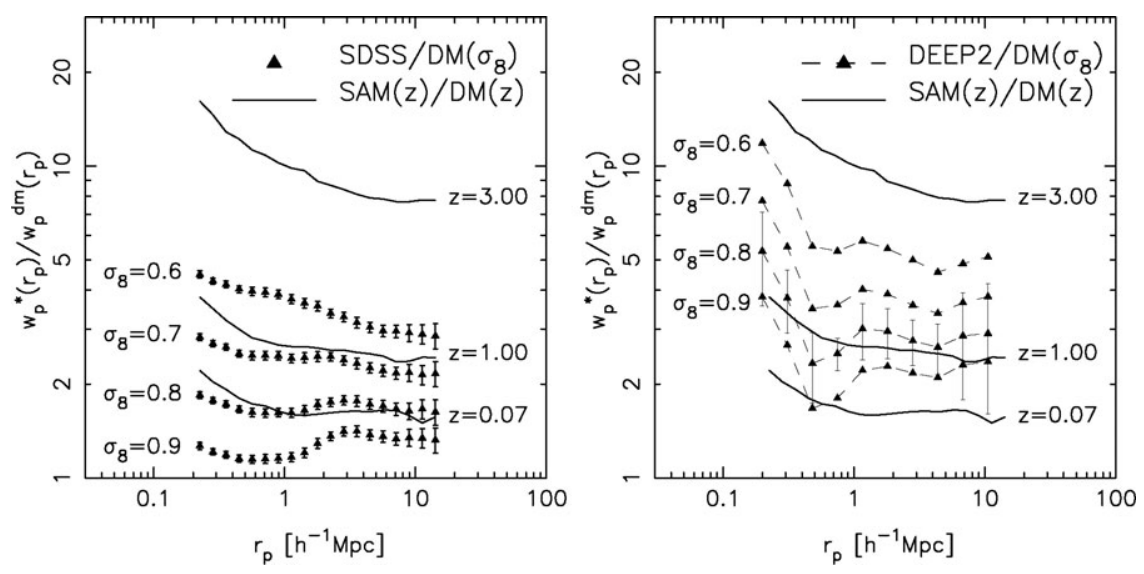

Figure 3. Triangles with error bars indicate bias as a function of scale for stellar mass in the SDSS (left panel) and in the DEEP2 (right panel) assuming the Millennium Simulation cosmology but with varying values (as labelled) for the present-day fluctuation amplitude $\sigma_{8}$. For $\sigma_{8}$ values other than 0.9 , projected dark matter autocorrelations at $z=0.07$ (for the SDSS) and $z=1$ (for the DEEP2) are approximated by the functions found in the Millennium Simulation at earlier times when the linear fluctuation amplitude matches that desired. Bias functions for stellar mass in the Guo et al. (2011) galaxy formation model (which assumes $\left.\sigma_{8}=0.9\right)$ at $z=0.07,1$ and 3 are shown by solid lines, labelled by redshift. For clarity, error bars are only shown in the right-hand panel in the case $\sigma_{8}=0.8$, and the triangles for each $\sigma_{8}$ are connected by a dotted line. Only for $\sigma_{8}=0.8$ or 0.7 is the bias function inferred from the data approximately as flat and featureless at the one-halo/two-halo transition as that measured in the model.

Theoretical predictions for these stellar mass bias functions for $z=0.07,1$ and 3 are shown for the galaxy formation model of G11 as solid lines in Figure 3. None of these curves show a feature at the one-halo/two-halo transition, but their slope increases significantly towards higher redshift. Comparing with the observational results, it is clear that the best agreement in amplitude occurs for $\sigma_{8}=0.8$ whereas the overall shape agrees best for somewhat lower amplitudes. It is remarkable that these results hold both for the SDSS at $z=0.07$ and for the DEEP2 at $z=1$. Hence the models appear to describe the overall evolution of the stellar mass autocorrelations surprisingly well, once the cosmology is corrected to a lower $\sigma_{8}$ value, similar to that indicated by most other recent analyses of CMB and other data (e.g., Komatsu et al. 2011).

\section{References}

Davis, M., Faber, S. M., Newman, J., et al. 2003, in Society of Photo-Optical Instrumentation Engineers (SPIE) Conference Series, Guhathakurta P., ed., Vol. 4834, pp. 161-172

Guo, Q., White, S., Boylan-Kolchin, et al. 2011, MNRAS, 413, 101

Komatsu, E., Smith, K. M., Dunkley, J., et al. 2011, ApJS, 192, 18

Li, C. \& White, S. 2009, MNRAS, 398, 2177

Li, C. \& White, S. 2010, MNRAS, 407, 515

Li, C., White, S., Chen, Y. M., et al. 2012, MNRAS, 419, 1557

York, D. G., Adelman, J., Anderson, J. E., Jr., et al. 2000, AJ, 120, 1579 